\title{
IMPULSE/RESPONSE FUNCTIONS OF INDIVIDUAL COMPONENTS OF FLOW-INJECTION MANIFOLDS
}

\author{
I.C. VAN NUGTEREN-OSINGA, M. BOS and W.E. VAN DER LINDEN* \\ Laboratory for Chemical Analysis-CT, University of Twente, P.O. Box 217, NL-7500 AE \\ Enschede (The Netherlands)
}

(Received 30th March 1988)

\section{SUMMARY}

The dispersion behaviour of the various individual parts making up a flow-injection manifold is often difficult to establish because it is virtually impossible to obtain the required very small injection and detection volumes. It is shown that it is possible, under suitable experimental conditions, to find the impulse/response function of each component by means of a deconvolution process of the response curves obtained with and without the part concerned. Once all the impulse/response functions have been established, the response function of any arrangement can be predicted by convoluting the impulse/response functions of all the individual parts involved. Convolution and deconvolution were done in the Fourier domain, by using a fast FT algorithm.

Many publications can be found in the literature dealing with theoretical aspects of sample dispersion in flow-injection systems. However, attention has been focussed almost exclusively on reaction/transport lines, e.g., straight tubes, coiled tubes, packed-bed reactors and single-bead-string reactors [1-6]. Although under certain simplifying conditions, analytical solutions of the general dispersion equation can be found, the approximations introduced in the derivations greatly restrict the usefulness of such solutions. This is the more serious because the initial and boundary conditions introduced do not conform, in general, to the experimental conditions normally prevailing in flowinjection analysis (FIA). Therefore, it has been suggested that analytical expressions for relevant parameters should be derived from numerical solutions of the diffusion-convection equation. This approach was first applied to FIA by Vanderslice et al. [7]. However, even straight tubes present great problems, and it is almost impossible to handle the situation for other component parts of the manifold such as mixing tees, connectors, membrane modules, etc. For this reason, it is proposed here to use an experimental approach and to evaluate the impulse/response functions of all individual component parts. Once these functions are known, the final result to be expected for any arrangement in FIA can be calculated by a mathematical convolution procedure. 
Another important application of impulse/response functions is their use in testing the performance of the individual components and so in the process of optimizing their construction. Normally, such impulse/response functions are determined by so-called "delta" injections (injection during an infinitesimally short period of time or injection of an infinitesimally small volume). On the FIA scale, this is virtually impossible and one has to accept a finite, but generally not exactly known, injection function. It will be shown that in that case the impulse/response function can be calculated by a deconvolution procedure, which is the inverse of convolution.

\section{THEORY}

The sample plug introduced in a flow-injection system can be characterized by means of an injection function $I(r, t)$. Because the concentration in the sample plug can normally be considered to be initially homogeneous over the cross-section (with radius $r$ ), this function simplifies to $I(t)$. If this sample plug is transported through a line to a detector, the function for the longitudinal dispersion of the sample entering the measuring cell, $M(t)$, can be described as the convolute of the injection function and the dispersion function of the transport line, $h(t)$, which is often called the impulse/response function. This convolution process is mathematically denoted here by the symbol *. So, $M(t)=I(t) * h(t)$. The reverse process, called deconvolution, will be denoted in the present text by the symbol //, e.g., $h(t)=M(t) / / I(t)$. The detector itself can also be characterized by an impulse/response function, $d(t)$, which gives the relation between the detector input and the output function, $O(t)$. Thus, $O(t)=I(t) * h(t) * d(t)$. If there are many elements contributing to the dispersion of the sample, the final output function is obtained by multiple convolution:

$$
O(t)=I(t) * h_{1}(t) * h_{2}(t) * h_{3}(t) \ldots * h_{n}(t) * d(t)
$$

It is important to note here that the solutions entering a consecutive dispersing element are assumed to be homogeneously mixed again in the radial direction, otherwise a parameter indicating the spatial distribution has to be included as well. This assumption is crucial in the following discussion, because it allows the order of convolution of the individual elements to be altered. Accordingly, measures have to be taken in the experimental stage to ensure this cross-sectional homogeneity. The impulse/response function of an individual element can be determined in the following way. In Fig. 1, a sample plug is introduced at the lefthand side and transported to the detector at the righthand side. The transport line is assumed to consist of two parts with impulse/response functions $h_{1}(t)$ and $h_{2}(t)$, respectively. The output function can then be described by

$$
f(t) \equiv O(t)=I(t) * h_{1}(t) * h_{2}(t) * d(t)
$$




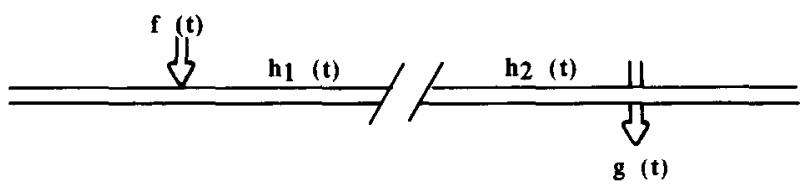

Fig. 1. Schematic diagram of the experimental approach.

If a new element is inserted between the two parts of the line and the impulse/ response function of this element is $h_{3}(t)$, then the new output function, $O^{\bullet}(t)$, is represented by

$g(t) \equiv O^{\bullet}(t)=I(t) * h_{1}(t) * h_{3}(t) * h_{2}(t) * d(t)$

Because the order of convolution can be altered without affecting the result, Eqn. (3) can be rewritten as

$g(t)=h_{3}(t) * I(t) * h_{1}(t) * h_{2}(t) * d(t)=h_{3}(t) * f(t)$

Thus the impulse/response function of the inserted element can be obtained by deconvolution of the two output functions obtained with and without this extra element, which eliminates the need for any knowledge of the functions $I(t), h_{1}(t)$ and $h_{2}(t)$ individually:

$h_{3}(t)=g(t) / / f(t)$

The mathematical processes of convolution and deconvolution must be done numerically. Therefore, the "input function", $f(t)$, and the "output" function, $g(t)$, have to be sampled at discrete intervals $f(i)$ and $g(i)$, respectively. The most straightforward procedure for convolution is direct calculation:

$g(k)=\sum_{i=\infty}^{k=\infty} f(i) h(k-i)$

For deconvolution, the successive values can be calculated by the following scheme:

$h(0)=g(1) / f(1)$;

$h(1)=[g(2)-f(2) h(0)] / f(1)$

$h(2)=[g(3)-f(2) h(1)-f(3) h(0)] / f(1)$ etc.

Other possible procedures are based on transformation techniques, such as the Laplace transform, $Z$-transform, and Fourier transform. An advantage of these transforms is that in the new domain, convolution is replaced by multiplication, while deconvolution corresponds to dividing the two transformed functions. Neither the numerical forward transformation, nor the subsequent division of the two discretized and transformed output functions present great mathematical problems and so, in principle, this approach is very attractive. 
The problem with the Laplace transform and $Z$-transform is, however, to achieve the inverse transformation back to the time domain. Although computer programs for the inverse transformation from the Laplace domain to the time domain are available, they are complicated [8] and require much computing capacity. If one is interested only in some characteristic values of a function such as the zero, first and second statistical moment (i.e., the area, the mean and the variance of the function, respectively), the back-transformation is not necessary, because the information needed can be obtained directly from the Laplace domain, as has been shown by van der Laan [9] (cf. [3] ). The $Z$-transform, which can be considered as a kind of discretized Laplace transform, has the same drawback and is not considered further in this paper. For these reasons, attention was focussed on the possibilities of the Fourier transform, which provides a frequency-domain analysis of time-dependent signals. Very fast and efficient algorithms are now available for this transformation (fast Fourier transform, FFT, algorithms), which are easily run even on a microcomputer. As in the case of the Laplace transform, convolution in the time domain is replaced by multiplication in the Fourier domain, and deconvolution by division [10]. A further advantage of using the frequency domain is that the contribution of noise can easily be reduced by omitting the higher frequencies. Because a very abrupt truncation of the frequencies considered can lead, on back-transformation, to so-called side-lobes in the time domain, a more gradual truncation is often chosen. The information about the peak shape in the time domain is included in the values of the amplitudes or Fourier coefficients in the frequency domain. This can be visualized either in Nyquist plots where the real part of the coefficient is plotted against the imaginary part or in Bode plots where the amplitudes are plotted against the corresponding frequency.

\section{EXPERIMENTAL}

All chemicals used were of analytical-reagent grade. A dye stock solution was prepared by dissolving $0.400 \mathrm{~g}$ of bromothymol blue in $25 \mathrm{ml}$ of ethanol and diluting to $100 \mathrm{ml}$ with $0.01 \mathrm{M}$ sodium tetraborate. Sample solutions were prepared by a 100 -fold dilution of this stock solution with $0.01 \mathrm{M}$ sodium tetraborate. The carrier solution was $2 \times 10^{-3} \mathrm{M}$ sodium tetraborate.

The manifold used is outlined in Fig 2. The peristaltic pump was a Gilson Minipuls-2. Polyethylene tubing was used. The injection valve was of the rotary type (Rheodyne) and was controlled by the computer. The injection volume was about $100 \mu \mathrm{l}$. Tubes ( $1.2 \mathrm{~mm}$ i.d., $30 \mathrm{~mm}$ long) packed with glass beads (diameter between 0.65 and $0.9 \mathrm{~mm}$ ) were used to achieve radial mixing wherever required, i.e., at the entrance to each element. The detector was a Zeiss PMQ-III variable-wavelength photometer equipped with an $80-\mu \mathrm{l}$ cell (Helma ). An Apple IIe computer was used for controlling the experiments and 


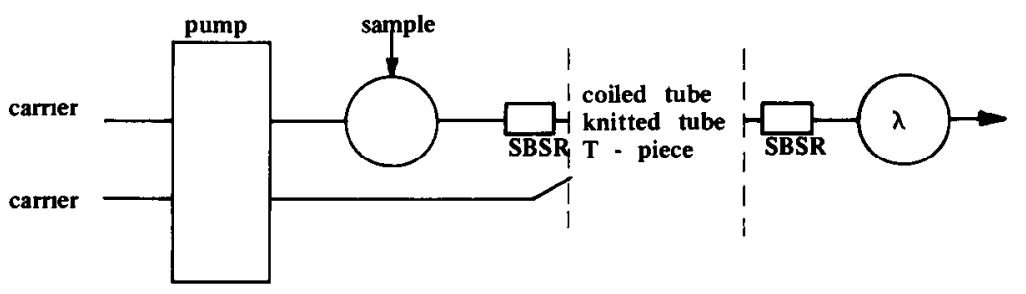

Fig. 2. Manifold for measuring the dispersion in tubes and T-pieces. The additional carrier stream is connected only when the manifold is used for measuring $T$-pieces.

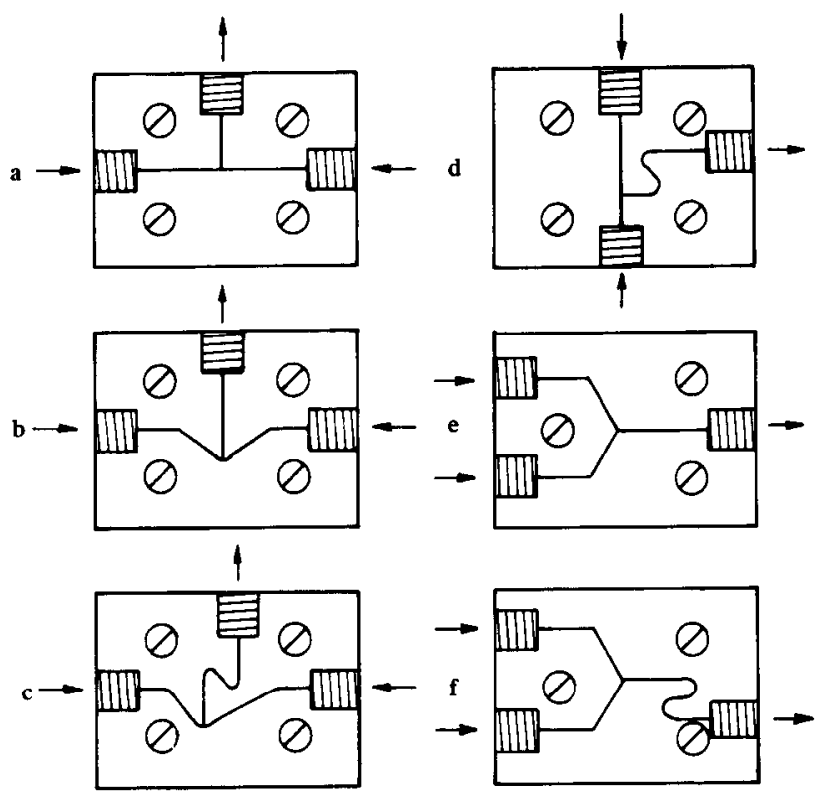

Fig. 3. Various types of mixing T-pieces.

for collecting the data. The response curves were sampled at a rate of $5 \mathrm{~s}^{-1}$. The modules tested consisted of $1 \mathrm{~m}$ of tubing ( $0.78 \mathrm{~mm}$ i.d.) coiled with coil diameters of 5,10 and $15 \mathrm{~mm}$, respectively; a fourth tube $(0.89 \mathrm{~mm}$ i.d. $)$ was "knitted" [11]. Response curves for the system with and without each module were measured at a constant pumping rate of $1.8 \mathrm{ml} \mathrm{min}^{-1}$ unless otherwise stated. Six different mixing T-pieces (Fig. 3) were tested. In order to make the flow rates for the samples passing the detector mutually comparable in the case of investigating the mixing $T$-pieces, the pumping rate used for measuring the input function was twice the pumping rate for measuring the response curve after insertion of the T-piece. 


\section{RESULTS AND DISCUSSION}

The determination of each impulse/response function started with the recording of the "basic" input function, $f(t)$, i.e., the signal obtained on injection of the dye sample without any additional element inserted in the transport line. Subsequently, the element of interest was inserted and again the signal was recorded. This signal was taken as the output function, $g(t)$. The subsequent mathematical procedure can be briefly summarized as follows:

$\begin{aligned} & f(t) \stackrel{\text { FFT }}{\longrightarrow} F(f) \\ & g(t) \stackrel{\text { FFT }}{\longrightarrow} G(f)\end{aligned} \rightarrow G(f) / F(f)=H(f) \stackrel{\text { inv. FFT }}{\longrightarrow} h(t)$

The FFT program used for the transformation was taken from Press et al. [12]. In Fig. 4, the responses with $[g(t)]$ and without $[f(t)]$ a coiled tube are depicted, together with the Bode and Nyquist plots of the transformed curve without the coiled tube. After the two curves in the frequency domain have
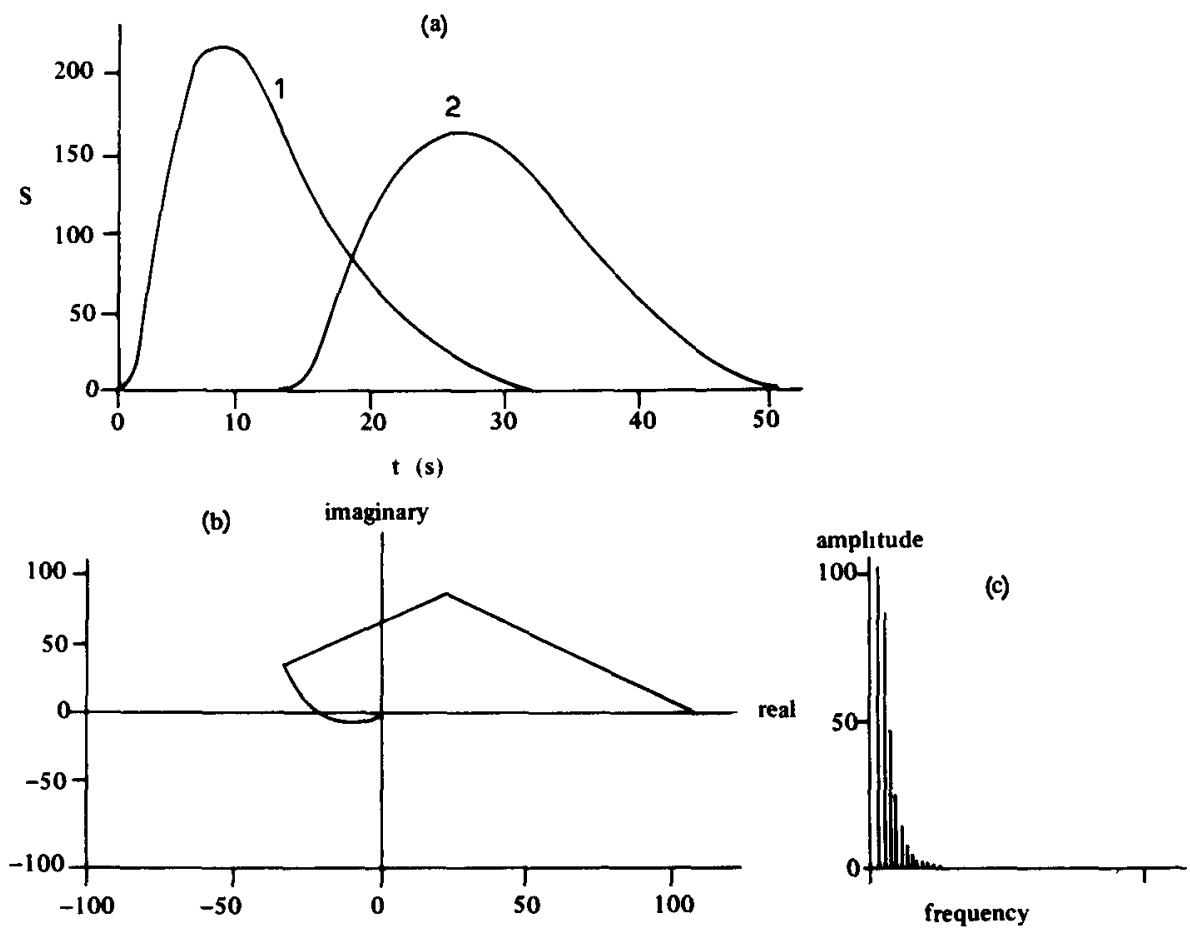

Fig. 4. (a) Response curves of the flow-injection system: (1) without extra coil inserted, $f(t)$; (2) with coil (coil diameter $5 \mathrm{~mm}$ ), $g(f)$. (b) Nyquist plot of the discrete Fourier transform $F(f)$ of the input function, $f(t)$. (c) Bode plot of the discrete Fourier transform $F(f)$. 
been divided, the Bode and Nyquist plots have the forms shown in Fig. 5. The values at higher frequencies are very unreliable, because they are the result of dividing two very small values each corrupted by noise. These values should therefore be omitted. Initially, only the first 16 frequencies were used in the back-transformation, which in fact corresponds to the use of a rectangular filter, but this leads to the appearance of spurious side-lobes (Fig. 6a). Consequently, a weighting function (filter) was chosen in such a way that the first five frequencies, which mainly determine the shape of the function in the time domain, are left unaffected whereas the amplitudes of the subsequent frequencies are reduced according to a logarithmically decreasing weighting function. As can be seen from Fig. 6(b), the general shape of the curve is not affected by this type of filtering but the spurious lobes have disappeared. In general, the choice of the filter is a matter of trial and error; a study of the optimal shape of the filter is in progress. Figure 7 shows the impulse/response functions of the three coiled tubes and the knitted tube. It can be seen that in all cases a small second peak appears after the major peak. This second peak cannot be attributed to the mathematical procedure, so it is not a computational artefact but has really to do with some physical phenomenon. This was also proved by

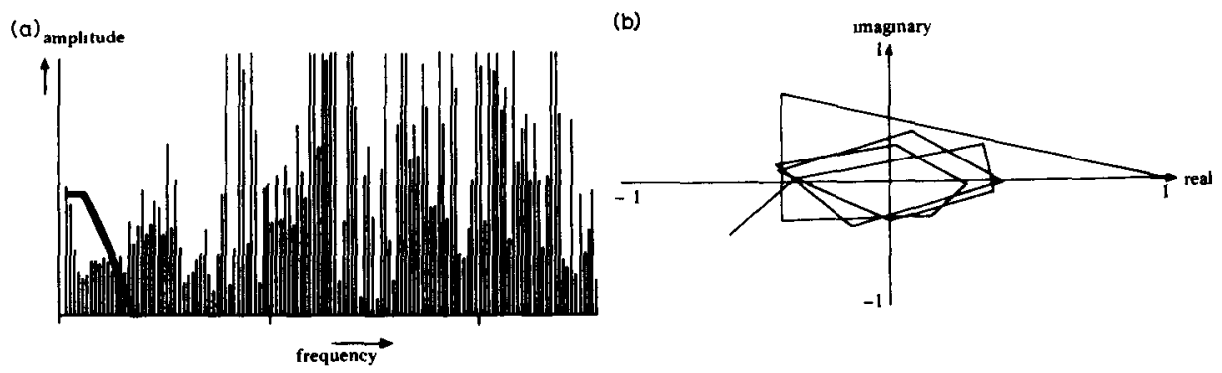

Fig. 5. (a) Bode plot of the discrete Fourier transform of the impulse/response function obtained by division of the Fourier transforms of the output function $g(t)$ and the input function $f(t)$; the thick line indicates the shape of the weighting factor used before inverse transformation to time domain. (b) Corresponding Nyquist plot for the first 16 frequencies.
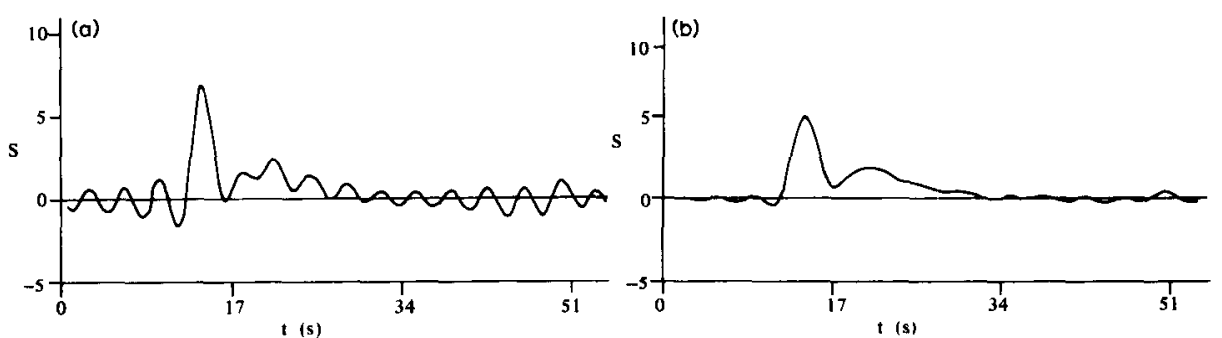

Fig. 6. Impulse/response curves for coiled tube ( $1 \mathrm{~m}$ long, $0.78 \mathrm{~mm}$ i.d., $5 \mathrm{~mm}$ coil diameter) at a flow rate of $1.8 \mathrm{ml} \mathrm{min}^{-1}$ : (a) rectangular filter; (b) logarithmic filter. 

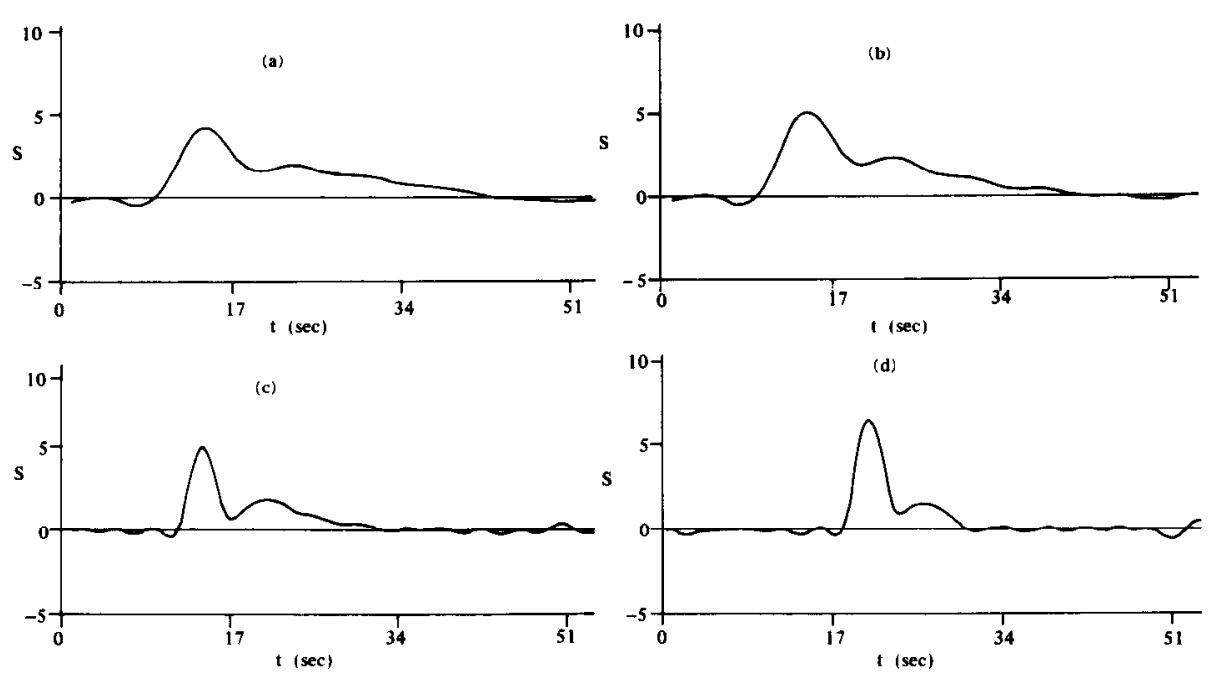

Fig. 7. Impulse/response curves for a tube $1 \mathrm{~m}$ long: (a) coil diameter $15 \mathrm{~mm}$; (b) coil diameter $10 \mathrm{~mm}$; (c) coil diameter $5 \mathrm{~mm}$; (d) "knitted". Internal diameter: (a-c) $0.78 \mathrm{~mm}$; (d) $0.89 \mathrm{~mm}$. Flow rate $1.8 \mathrm{ml} \mathrm{min}^{-1}$.

an independent curve-fitting procedure. When the output curves were fitted to a tanks-in-series model, the curve obtained without the extra coil gave a good fit to the equation

$g(t)=(-1 / \tau)\left[(t / \tau)^{n-1} /(n-1) !\right] \exp (-t / \tau)$

where $n$ is the number of tanks, and $\tau$ is the residence time in one tank. However, when an attempt was made to fit the output curves of the system provided with the extra coil to the same simple model, no acceptable fit could be obtained, but a good fit was obtained with the following model:

$$
\begin{aligned}
g(t)= & a(-1 / \tau)\left[(t / \tau)^{n_{1}-1} /\left(n_{1}-1\right) !\right] \exp (-t / \tau) \\
& +(1-a)(1 / \tau)\left[(t / \tau)^{n_{2}-1} /\left(n_{2}-1\right) !\right] \exp (-t / \tau)
\end{aligned}
$$

when $n_{1} \approx 3, n_{2} \approx 5$ and $a \approx 0.6$. This means that the dispersion behaviour in the -coil can be described by two parallel tanks-in-series models with different numbers of tanks and so a different hold-up time. The experiment was repeated at various flow rates. It was found that the peak maxima of the two peaks appearing in the impulse/response functions are linearly dependent on the inverse flow rate; but the most interesting fact is that the first peak shifts at half the speed of the second peak, i.e., $\tau \approx L / 2 \bar{v}$ and $L / \bar{v}$, respectively, where $L$ is the coil length and $\bar{v}$ is the mean flow rate. This suggests that the major, faster peak can be attributed to more convective behaviour, whereas diffusion is involved in the case of the second, slower and smaller, peak. Hitherto, such 


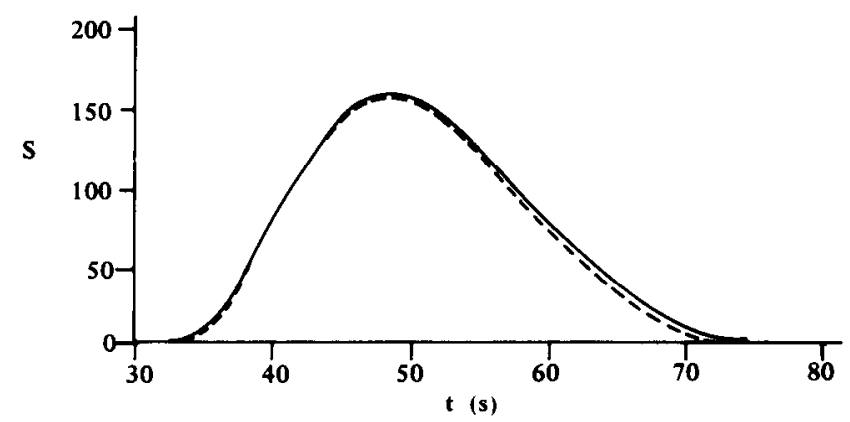

Fig. 8. Response curve obtained with two coils in series: (-) calculated; (- - ) experimental.

double humped peaks have only been observed under very strictly laminar flow conditions with a very small injection volume and a detector with a small effective volume [3]. In more usual arrangements, with larger injection and detector volumes, this dispersion effect is not observed and is apparently obscured by the convoluting properties of the various components of the system. Although a study of the theoretical aspects of dispersion behaviour was not the aim of the present work, this example illustrates the power of the straightforward method proposed here for the determination of the impulse/response function.

From Fig. 7, it is obvious that the "knitted" reactor provides the best performance with respect to reduction of dispersion when compared to the various coiled tubes. As for the various T-pieces, little mutual difference was found with respect to dispersion. The extra bends in the T-pieces shown in Fig. 3(c, $\mathrm{d}, \mathrm{f}$ ) did not produce any improvement and led only to a slightly longer residence time. In fact, the T-piece (a), which is the easiest to manufacture, appeared to be slightly preferable. To show that the procedure described above can also be used for the prediction of response curves of more complex systems, the impulse/response curves of two coils and the input function obtained without any coil were convoluted. As can be seen from Fig. 8, there is fairly good agreement between the calculated and experimental curves.

\section{Conclusions}

It has been shown that it is possible to determine the impulse/response function of individual parts of flow-injection systems by a mathematical deconvolution procedure. Based on such curves, an unbiased choice can be made as to which part out of a series of similar parts exhibits the best performance. Furthermore, the result of any arrangement of modules can be predicted once each part has been characterized. Work on the characterization of modules such as membrane modules for gas diffusion is in progress.

The authors thank H.M.M. de Jong for his help with the implementation of the FFT program and G. van Straten for helpful discussions on some theoret- 
ical aspects. These investigations were supported (in part) by the Netherlands Foundation for Chemical Research (SON) with financial aid from the Netherlands Technology Foundation.

\section{REFERENCES}

1 J. Ruzicka and E.H. Hansen, Flow Injection Analysis, 2nd edn., Wiley, New York, 1988.

2 M. Valcárcel and M.D. Luque de Castro, Flow-Injection Analysis: Principles and Applications, Horwood, Chichester, 1987.

3 J.M. Reijn, W.E. van der Linden and H. Poppe, Anal. Chim. Acta, 114 (1980) 105; 123 (1981) $229 ; 126$ (1981) 1 ; 145 (1983) 59.

4 R. Tijssen, Anal. Chim. Acta, 114 (1980) 71.

5 J.H.M. van den Berg, R.S. Deelder and H.G.M. Egberink, Anal. Chim. Acta, 114 (1980) 91.

6 J.T. Vanderslice, A.G. Rosenfeld and G.R. Beecher, Anal. Chim. Acta, 179 (1986) 119.

7 J.T. Vanderslice, K.K. Stewart, A.G. Rosenfeld and D.J. Higgs, Talanta, 28 (1981) 11.

8 S.D. Kolev and E. Pungor, Anal. Chim. Acta, 185 (1986) 315; 194 (1987) 61.

9 E.T. van der Laan, Chem. Eng. Sci., 7 (1955) 187.

10 D.L. Massart, B.G.M. Vandeginste, S.N. Deming, Y. Michotte and L. Kaufman, Chemometrics: A Textbook, Elsevier, Amsterdam, 1987, Chap. 15.

11 U. Neue and H. Engelhardt, Chromatographia, 15 (1982) 403.

12 W.H. Press, B.P. Flannery, S.A. Teukolsky and W.T. Vetterling, Numerical Recipes, Cambridge University Press, Cambridge, 1986. 